\title{
ATMOSPHERIC PENETRATION OF EXHAUST PLUMES UNDER RAREFIED FLOW CONDITIONS*
}

\author{
BY \\ HOWARD R. BAUM ${ }^{1}$ AND APOSTOLOS E. GERMELES ${ }^{2}$ \\ Aerodyne Research, Inc., Burlington, Mass.
}

\begin{abstract}
The interaction of a low-density atmosphere with the exhaust gases produced by the steady firing of a rocket motor moving at hypersonic speed is studied using the kinetic theory of gases. The Krook collision model is employed in conjunction with a simple representation of the exhaust gas distribution to derive analytical and numerical solutions for the atmospheric gas density as it penetrates the rocket plume. The atmosphere gas is composed of an initially unscattered beam, which is attenuated as it collides with the much denser exhaust plume, and a scattered component, which is convected away from the motor by the expanding jet. These processes are illustrated by the computed results for both an axially symmetric and a three-dimensional flow.
\end{abstract}

1. Introduction. The flow pattern set up by the interaction of an exhaust plume generated by the continuous firing of a rocket motor and a tenuous atmosphere has been intensively studied in recent years. Most of this work has focused on those domains of motor thrust and operating altitude permitting this interaction to be described on the basis of continuum fluid mechanics. At altitudes sufficiently high for the ambient mean free path to become larger than the continuum interaction length scale, the analysis of such a flow must be based on the kinetic theory of gases. Under these conditions, the length scale associated with the atmospheric penetration of the exhaust plume is $\sqrt{ } A / \mathrm{Kn}$, where $A$ is the nozzle exit area and $\mathrm{Kn}$ is the Knudsen number based on $\sqrt{ } A$ and the mean free path, referred to average exit conditions (see [8]). This scale is, typically, much larger than the dimensions of the vehicle carrying the motor, since $\mathrm{Kn} \ll 1$. The presence of the vehicle itself may thus be ignored in analyzing phenomena on this scale.

When the penetration scale is small compared with the ambient mean free path, the response of the atmosphere to the presence of the plume is decoupled from the process by which the atmosphere modifies the vacuum plume expansion. This situation is appropriate to moderate-sized engines, such as those employed for attitude control or maneuvering purposes in the upper atmosphere. The spatial separation of these two phenomena has been exploited by Brook and Hamel [4] in their study of a spherical source interacting with a stationary background gas. Baum [3] considered the expansion

\footnotetext{
* Received May 23, 1974. This work was supported by the Army Ballistic Missile Defense Agency under contract Number DAHC 60-69-C-0035.

1 Present address: National Bureau of Standards, Washington, D. C. 20234, U. S. A.

${ }^{2}$ Present address: Technical Staff, Corporate Research, Cabot Corp., Billerica, Mass. 01821, U. S. A.
} 
of a transient exhaust plume into a rarefied atmosphere by invoking equivalent assumptions about the scales associated with the interactions occurring in that problem.

The present work is concerned with the penetration of a hypersonic ambient stream into a steady-state exhaust plume under the conditions stated above. The exhaust gas properties are computed from a simple model, proposed by Hill and Draper [7], of the continuum expansion of a jet into a vacuum. This expanding jet serves as a diffuse scatterer of ambient molecules. The scattering is represented mathematically by the Krook collision integral. This formulation leads to an analytically interpretable and computationally tractable integral equation for the ambient density distribution. The molecular distribution function is described analytically in terms of the density. The distribution function is bimodal, with the unscattered molecules peaked about the uniform free-stream velocity, and the scattered gas convected with the local macroscopic exhaust velocity. An asymptotic, analytical solution to the integral equation is obtained. This result is used, in conjunction with direct numerical solutions of the integral equation, to obtain illustrative flow patterns for both axially symmetric and nonsymmetric configurations.

2. Mathematical formulation. The formulation of the model will now be considered in detail. The penetration scale is assumed to be sufficiently small compared with the ambient mean free path for collisions between ambient molecules to be ignored. The kinetic equation for the ambient, molecular distribution function $f(\mathbf{x}, \mathbf{v})$ is a modified version of that given by Gross and Krook [5]:

$$
\begin{aligned}
\mathbf{v} \cdot \frac{\partial f}{\partial \mathbf{x}} & =\omega(n \Phi-f), \\
\omega & =n_{e} \sigma\left|\mathbf{u}_{\infty}-\mathbf{u}_{M}\right|, \\
\Phi & =\left(\frac{m}{2 \pi k T_{\iota}}\right)^{3 / 2} \exp \left\{-\frac{m}{2 k T_{e}}\left(\mathbf{v}-\mathbf{u}_{e}\right)^{2}\right\}, \\
n(\mathbf{x}) & =\int f d^{3} v .
\end{aligned}
$$

Here $n(\mathbf{x})$ is the ambient number density, and $n_{e}, \mathbf{u}_{\epsilon}, T_{\epsilon}$ are, respectively, the exhaust gas number density, macroscopic velocity, and temperature. The maximum velocity of the exhaust gas is $\mathbf{u}_{M}$, while the cross-section $\sigma$, entering the expression for the collision frequency $\omega$, is an effective momentum transfer cross-section. The relation between the momentum transfer between species computed from (1) and that given by Gross and Krook [5] is explained in Baum [3]. The mean relative speed is taken to be that between $\mathbf{u}_{M}$ and the ambient wind velocity $\mathbf{u}_{\infty}$ because the exhaust and ambient speed ratios, $W_{c}$ and $W_{\infty}$, are large under the conditions of interest.

The exhaust properties are assumed in a modified version of the form devised by Hill and Draper [7]. The forms adopted are equivalent to the Hill-Draper model far from the engine, but take on the prescribed nozzle exit values at $\mathbf{x}=0$. The relevant equations are

$$
\frac{n_{e}}{n_{0}}=\frac{B A_{e x i t} \exp \left\{-\Lambda_{\infty}^{2}(1-\cos \theta)^{2}\right\}}{r^{2}+B A_{e x i t} \exp \left\{-\Lambda_{\infty}^{2}(1-\cos \theta)^{2}\right\}},
$$




$$
\begin{gathered}
T_{e} / T_{0}=\left(n_{e} / n_{0}\right)^{\gamma-1}, \quad \mathbf{u}_{e}=u_{e} \hat{r}, \\
\frac{u_{e}{ }^{2}}{2}+\frac{\gamma}{\gamma-1} \frac{k T_{e}}{m_{e}}=\frac{u_{M}{ }^{2}}{2}=\frac{u_{0}^{2}}{2}\left[1+\frac{2}{\gamma-1} \frac{1}{M_{0}^{2}}\right] .
\end{gathered}
$$

The parameters $B$ and $\Lambda_{\infty}$ are given in terms of exit properties by

$$
\begin{aligned}
& B=\left(\Lambda_{\infty} / \pi^{3 / 2}\right)\left(u_{0} / u_{M}\right), \\
& \Lambda_{\infty}=\frac{1}{\sqrt{ } \pi}\left\{1-\frac{u_{0}}{u_{M}}\left(1+\frac{1}{\gamma M_{0}^{2}}\right)\right\}^{-1} .
\end{aligned}
$$

The nozzle exit number density $n_{0}$, speed $u_{0}$, temperature $T_{0}$, Mach number $M_{0}$, and the ratio of specific heats $\gamma$ are determined by the engine parameters. The exhaust properties are expressed in a spherical, polar coordinate system, centered about the unit thrust vector $\hat{t}$. The polar angle is $\theta$, and the azimuthal angle $\phi$ is measured from the plane containing the wind and thrust vectors. The wind vector makes an angle $\psi$ with the thrust vector. Thus (see Fig. 1)

$$
\begin{aligned}
\mathbf{x} & =r(\hat{t} \cos \theta+\hat{\jmath} \sin \theta \cos \phi+\hat{k} \sin \theta \sin \phi) \equiv \hat{r} r \\
\mathbf{u}_{\infty} & =u_{\infty}(\hat{t} \cos \psi+\hat{\jmath} \sin \psi), \\
\mathbf{v} & \equiv V \hat{v}=V(\cos \xi \hat{t}+\sin \xi \cos \eta \hat{\jmath}+\sin \xi \sin \eta \hat{k}) .
\end{aligned}
$$

All quantities with a caret superscript are unit vectors. The system (1) through (3) must be supplemented by the boundary condition that, far from the plume, the flow is uniform:

$$
\begin{gathered}
\lim _{r \rightarrow \infty} f(\mathbf{x}, \mathbf{v})=n_{\infty}\left(\frac{m}{2 \pi k T_{\infty}}\right)^{3 / 2} \exp \left\{\frac{-m}{2 k T_{\infty}}\left(\mathbf{v}-\mathbf{u}_{\infty}\right)^{2}\right\} \equiv f_{\infty}, \\
W_{\infty}=u_{\infty} /\left(k T_{\infty} / m\right)^{1 / 2}
\end{gathered}
$$

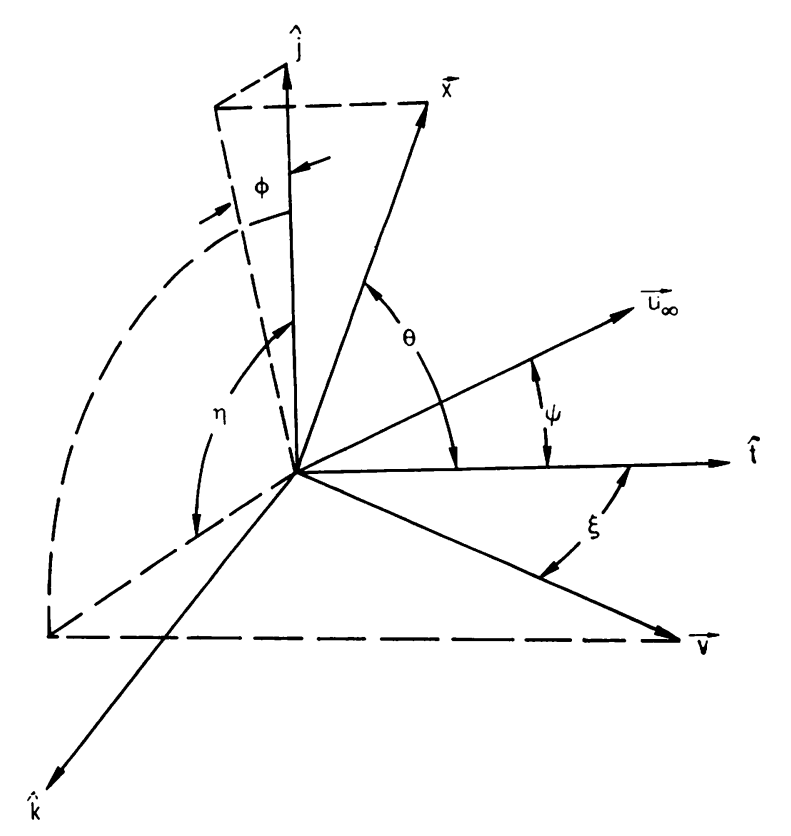

FIG. 1. Coordinate system geometry. Nozzle exit at origin. 
Here $n_{\infty}, \mathbf{u}_{\infty}$, and $T_{\infty}$ are, respectively, the ambient number density, velocity, and temperature far from the plume.

The representation of the exhaust gas displayed in (2) and (3) describes the expansion into vacuum of a continuum jet plume. The motion is isentropic and isoenergetic. The density distribution (and, hence, each displayed fluid quantity) satisfies the inviscid equations of motion asymptotically, far from the nozzle exit. The angular dependence of the asymptotic density distribution is chosen as an empirical curve fit to numerical methods of characteristic solutions for a variety of engines. The expressions for the parameters $B$ and $\Lambda_{\infty}$ ensure global conservation of mass, momentum, and energy.

The continuum description of the exhaust gas ultimately breaks down as the plume expands. The rarefaction process has been studied by Grundy [6], who shows that, except at large angles $\theta$, with respect to the thrust axis, the density and velocity are given by the continuum solution. The radial component af the pressure tensor, on the other hand, does not continue to decrease isentropically, but levels off at a finite, "frozen" asymptote. The Mach number corresponding to the freezing temperature is typically very large, on the order of $15-25$ for most angles $\theta$. When the local Mach number is large, however, the interaction between ambient and exhaust gases is nearly independent of the local plume temperature, as shown by the asymptotic solution obtained in Sec. 4 .

The next step in the calculation is the construction of the fundamental integral equation for $n(\mathbf{x})$. Following Anderson [1], (1) may be written in characteristic form as

$$
V \frac{d}{d s} f(\mathbf{x}+\hat{v} s)=(\mathbf{x}+\hat{v} s) n\{\Phi(\mathbf{x}+\hat{v} s)-f\}, \quad \mathbf{r}=\mathbf{x}+\hat{v} s .
$$

This describes the evolution of $f$, at any point $\mathbf{r}$, a distance $s$ along a ray through the field point $\mathbf{x}$, in the direction $\hat{v}$ (see Fig. 2). Now integrate (5) from minus infinity to the

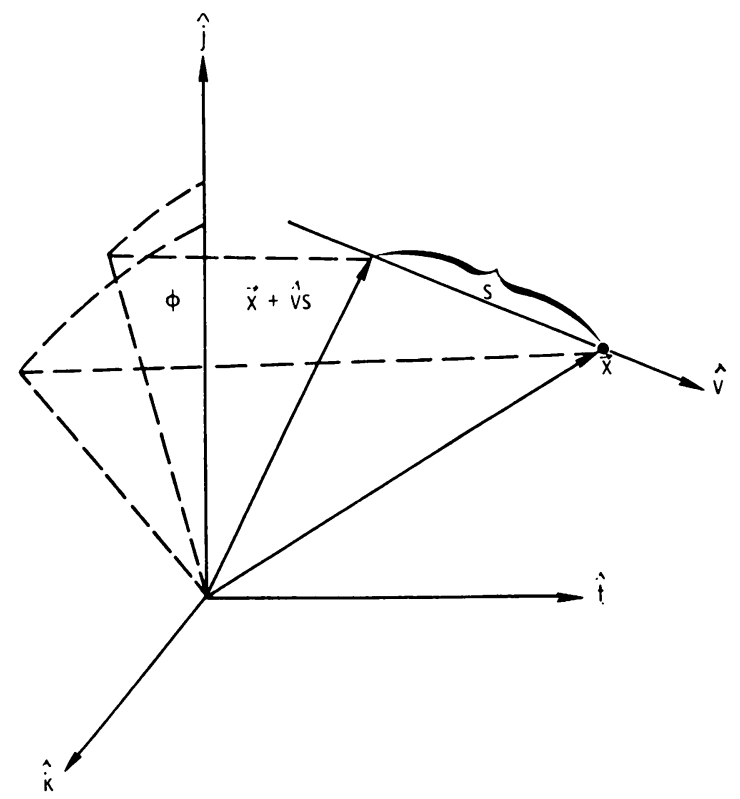

FIG. 2. Characteristic ray geometry. Molecules at $\mathbf{x}$ arrive from all directions $\hat{v}$. The probability of arrival depends upon the distance $s$ from the point of last collision. 
field point $s=0$, applying the boundary condition represented by (4). The result is

$$
\begin{aligned}
f & =f_{\infty} \exp (-\tau / v)+\frac{1}{v} \int_{-\infty}^{0} d s^{\prime} \omega\left(\mathbf{x}+\hat{v} s^{\prime}\right) \Phi\left(\mathbf{x}+\hat{v} s^{\prime}\right) \exp \left(-\tau^{*} / V\right) n\left(\mathbf{x}+\hat{v} s^{\prime}\right), \\
\tau & =\int_{-\infty}^{0} \omega(\mathbf{x}+\hat{v} s) d s, \\
\tau^{*} & =\int_{e^{\prime}}^{0} \omega(\mathbf{x}+\hat{v} s) d s, \\
\Phi\left(\mathbf{x}+\hat{v} s^{\prime}\right) & =n_{e}\left(\frac{m}{2 k T_{e}}\right)^{3 / 2} \exp \left\{-\frac{m}{2 k T_{e}}\left[V-u_{e} \cos \alpha\right]^{2}-\frac{1}{2} W_{e}^{2}\left[1-\cos ^{2} \alpha\right]\right\}, \\
\cos \alpha & \equiv \hat{v} \cdot \hat{r}=\frac{[\cos \xi \cos \theta+\sin \xi \sin \theta \cos (\eta-\phi)] r+s^{\prime}}{\left\{r^{2}+\left(s^{\prime}\right)^{2}+2 r s^{\prime}[\cos \theta \cos \xi+\sin \theta \sin \xi \cos (\eta-\phi)]\right\}^{1 / 2}} .
\end{aligned}
$$

The argument of $n_{e}, u_{e}$, and $T_{e}$ in (6) is $\mathbf{x}+\hat{v} s^{\prime}$. Note that the $V$ dependence is now displayed explicitly, since $\hat{v}$ depends only on the angle variables $\xi$ and $\eta$. The integral equation follows from (6), by using the definition of $n$ in terms of the distribution function $f$ :

$$
n=\int_{0}^{\pi} \sin \xi d \xi \int_{0}^{2 \pi} d \eta \int_{0}^{\infty} v^{2} d v f
$$

Thus

$$
\begin{array}{r}
\frac{n}{n_{\infty}}=\frac{1}{2 \pi} \int_{0}^{\pi} \sin \xi d \xi \int_{0}^{2 \pi} d \eta\left\{H_{4}\left(W_{\infty} \cos \beta ; \tau\left(\frac{m}{k T_{\mathrm{e}}}\right)^{1 / 2}\right) \exp \left[-\frac{1}{2} W_{\infty}^{2}\left(1-\cos ^{2} \beta\right)\right]\right. \\
+\int_{-\infty}^{0} d s^{\prime} W\left(\frac{m}{k T_{\mathrm{e}}}\right)^{1 / 2} H_{3}\left(W_{\mathrm{c}} \cos \alpha ; \tau^{*}\left(\frac{m}{k T_{\mathrm{e}}}\right)^{1 / 2}\right) \\
\left.\cdot \exp \left[-\frac{1}{2} W_{\mathrm{e}}^{2}\left(1-\cos ^{2} \alpha\right)\right] \frac{n}{n_{\infty}}\left(\mathbf{x}+\hat{v} s^{\prime}\right)\right\},
\end{array}
$$

$\cos \beta=\cos \xi \cos \psi+\sin \xi \sin \psi \cos \eta$.

Again, all exhaust properties in (7) are functions of $\mathbf{x}+\hat{v} s^{\prime}$. The functions $H_{n}(p, q)$ are defined by

$$
H_{n}(p, q)=\frac{-1}{(2 \pi)^{1 / 2}} \int_{0}^{\infty} d V V^{n-2} \exp \left\{-\frac{1}{2}(V-p)^{2}-q / V\right\} .
$$

Anderson and Macomber [2] contains a detailed analysis of this function, together with tables for $n=1,2$, 3. Eq. (7) determines the spatial density distribution of the atmosphere as it penetrates the plume core, while (6) expresses the distribution function in terms of $n$. In this form, the equations are too complicated to be useful. However, by utilizing the fact that, in many cases of interest, both $W_{e}$ and $W_{\infty}$ are large, (6) and (7) can be reduced to a tractable form.

3. The hypersonic limit. Consider the first term in the integral (7). For large $W_{\infty}$, the exponential factor suggests that the integrand, considered as a function of $\xi$, is strongly peaked about $\cos \beta^{2}=1$. Examination of the $H_{n}$ functions reveals that $H_{n}$, considered as a function of $p$, is exponentially small for large negative $p$ and propor- 
tional to $p^{n-2}$ for large positive $p$. Since the dependence of $H_{n}$ on $W_{\infty}$ for large positive $p$ is weak, apart from a purely multiplicative factor $W_{\infty}{ }^{n-2}$, the dominant contribution to the $\xi$ integration should come from the neighborhood of $\cos \beta=+1$.

To proceed formally from these ideas, it is more convenient to do the $\xi$ integral first, and employ the variable $x=\cos \xi$. The integral under consideration assumes the form:

$I=\int_{-1}^{1} d x H_{4}(x) \exp \left\{-\frac{1}{2} W_{\infty}^{2} g(x)\right\}, \quad g(x)=1-\left\{x \cos \psi+\left(1-x^{2}\right)^{1 / 2} \sin \psi \cos \eta\right\}^{2}$.

Applying the method of steepest descent to (8), the saddle point is at

$$
x_{0}= \pm \frac{\cos \psi}{\left(1-\sin ^{2} \psi \sin ^{2} \eta\right)^{1 / 2}}, \quad \cos \beta\left(x_{0}\right)= \pm\left(1-\sin ^{2} \psi \sin ^{2} \eta\right)^{1 / 2} .
$$

Both saddle points are real, but the contribution from the one associated with the minus sign is exponentially smaller than that associated with the positive value of $\cos \beta$, and is henceforth ignored. The integral $I$ is now readily evaluated as

$$
\begin{gathered}
I \cong \frac{2}{W_{\infty}}\left(\frac{\pi}{g^{\prime \prime}\left(x_{0}\right)}\right)^{1 / 2} \exp \left(-\frac{1}{2} W_{\infty}^{2} g\left(x_{0}\right)\right) H_{4}\left(W_{\infty} \cos \beta ; \tau\left(x_{0}\right) \frac{m}{k T_{\infty}}\right), \\
g\left(x_{0}\right)=\sin ^{2} \psi \sin ^{2} \eta, \quad g^{\prime \prime}\left(x_{0}\right)=\frac{2}{1-x_{0}^{2}}\left(1-\sin ^{2} \eta \sin ^{2} \psi\right), \\
x_{0}=\frac{\cos \psi}{\cos \beta}, \quad \cos \beta=\left(1-\sin ^{2} \psi \sin ^{2} \eta\right)^{1 / 2}
\end{gathered}
$$

Using the result (9), the integration over $\eta$ may be performed. The required integral is then

$$
J=\frac{1}{2 \pi} \int_{0}^{2 \pi} I d \eta
$$

Examination of this expression when $W_{\infty} \sin \psi \cdot \gg 1$ (i.e., the undisturbed flow is neither parallel nor antiparallel to the thrust vector) shows that the dominant contribution comes from the saddle point at $\eta=0$. The apparent saddle point at $\eta=\pi$ is spurious, with the result that

$$
J=\frac{1}{W_{\infty}^{2}} H_{4}\left(W_{\infty},\left(\frac{m}{k T_{\infty}}\right)^{1 / 2} \tau\right), \quad \tau \equiv \tau(\xi=\psi ; \eta=0) .
$$

A separate calculation when $W_{\infty} \sin \psi$ is $O(1)$ (but $W_{\infty} \gg 1$ ) yields the same result for $J$. Physically, (10) states that the overwhelming majority of the unscattered ambient molecules are aligned with the uniform wind upstream of the plume. A similar analysis (the details of which are presented in Appendix A) may be applied to the homogeneous term in (7). The hypersonic limit of (7) then becomes

$$
\begin{gathered}
\frac{n}{n_{\infty}}(y)=\frac{1}{W_{\infty}^{2}} H_{4}\left(W_{\infty}, q_{\infty}\right)+\int_{0}^{\nu} d y^{\prime} K\left(y^{\prime}, y\right) \frac{n}{n_{\infty}}(y), \\
K\left(y, y^{\prime}\right)=\left[1+\left(\frac{u_{M}}{u_{\infty}}\right)^{2}-\frac{2 u_{M}}{u_{\infty}} \cos \beta^{*}\right]^{1 / 2}\left(\frac{y^{\prime}}{y}\right)^{2} \frac{u_{\infty}}{u_{e}}\left(y^{\prime}\right) \frac{n_{e}}{n_{0}}\left(y^{\prime}\right) \cdot \frac{1}{W_{e}} H_{3}\left(W_{e}, q_{e}\right) .
\end{gathered}
$$


Here:

$$
\begin{gathered}
W_{c}=W_{e}\left(y^{\prime}\right), \quad q_{c}=q_{e}\left(y^{\prime}\right), \quad \cos \beta^{*}=\cos \theta \cos \psi+\sin \theta \sin \psi \cos \phi, \\
y=r(B A)^{-1 / 2}(\mathrm{Kn}), \quad(\mathrm{Kn})^{-1}=n_{0} \sigma(B A)^{1 / 2}, \\
q_{\infty}=\left(\frac{m}{k T_{\infty}}\right)^{1 / 2} \tau(\mathrm{x}+\hat{a} s), \quad q_{\bullet}=\left(\frac{m}{k T_{\mathrm{c}}}\right)^{1 / 2} \tau^{*}\left(\mathrm{x}+\hat{r} s^{\prime}\right) .
\end{gathered}
$$

The inhomogeneous term in (11) contains the variable $q_{\infty} \equiv\left(m / k T_{\infty}\right)^{1 / 2} \tau(\mathbf{x}+\cdot \hat{a} s)$, where $\hat{a}$ is a unit vector in the direction of the undisturbed wind. This quantity is directly proportional to the number of collisions experienced by a molecule traveling in a straight line from infinity to the point in question in the direction of the undisturbed wind. Written explicitly, it has the form:

$$
\begin{gathered}
\frac{q_{\infty}}{W_{\infty}}=y^{-1} \int_{-\infty}^{0} d t \frac{\left[1+\left(\frac{u_{M}}{u_{\infty}}\right)^{2}-2 \frac{u_{M}}{u_{\infty}} \cos \delta\right]^{1 / 2} \exp \left\{-\Lambda_{\infty}{ }^{2}(1-\cos \mu)^{2}\right\}}{1+t^{2}+2 t \cos \beta^{*}+\frac{\mathrm{Kn}^{2}}{y^{2}} \exp \left\{-\Lambda_{\infty}{ }^{2}(1-\cos \mu)^{2}\right\}}, \\
\cos \mu=\frac{\cos \theta+t \cos \psi}{\left(1+t^{2}+2 t \cos \beta^{*}\right)^{1 / 2}}, \quad \cos \delta=\frac{\cos \beta^{*}+t}{\left(1+t^{2}+2 t \cos \beta^{*}\right)^{1 / 2}} .
\end{gathered}
$$

As $y \rightarrow \infty$, the number of collisions vanishes and the inhomogeneous term in (11) approaches unity. As $y \rightarrow 0$, the number of collisions experienced becomes very large and the inhomogeneous term vanishes exponentially. The number of collisions also becomes large along rays near $\phi=0$ and $\theta=\psi$, since the molecule has then had to traverse the near singularity in exhaust gas density at the origin. Physically, the ray on the opposite side of the nozzle exit from the wind is shielded from the ambient molecules for finite distances $r$ from the exit. The nature of $q_{\infty}$ near this singular ray (corresponding to $\cos \beta^{*}=1$ ) may be analyzed as follows: the behavior is dominated by the singularity at $\cos \beta^{*}=1$, as $y$ approaches infinity or Kn vanishes. Thus, the quantities $\cos \mu$ and $\cos \delta$ in (13) may be replaced by their values at $\cos \beta^{*}=1$, i.e.,

$$
\begin{gathered}
\cos \mu \cong-\cos \psi, 1+t \leq 0, \quad \cos \mu \cong \cos \psi, 1+t>0, \\
\cos \delta \cong-1,1+t \leq 0, \quad \cos \delta \cong 1,1+t>0 .
\end{gathered}
$$

Upon inserting these values into (13) the integral for $q_{\infty}$ may be evaluated explicitly as

$$
\begin{gathered}
\frac{q_{\infty}}{W_{\infty}} \cong \frac{1}{y}\left\{\left(1+\frac{u_{M}}{u_{\infty}}\right) \exp \left[-\Lambda_{\infty}^{2}(1+\cos \psi)^{2}\right]\left[\frac{1}{a} \tan ^{-1}\left(\frac{-1+\cos \beta^{*}}{a}\right)+\frac{\pi}{2 a}\right]\right. \\
\left.+\left|1-\frac{u_{M}}{u_{\infty}}\right| \exp \left[-\Lambda_{\infty}{ }^{2}(1-\cos \psi)^{2}\right]\left[\frac{1}{b} \tan ^{-1}\left(\frac{\cos \beta^{*}}{b}\right)-\tan ^{-1}\left(\frac{-1+\cos \beta^{*}}{b}\right)\right]\right\}, \\
a=\left\{1-\cos ^{2} \beta^{*}+\left(\frac{\mathrm{Kn}}{y}\right)^{2} \exp \left[-\Lambda_{\infty}^{2}(1+\cos \psi)^{2}\right]\right\}^{1 / 2}, \\
b=\left\{1-\cos ^{2} \beta^{*}+\left(\frac{\mathrm{Kn}}{y}\right)^{2} \exp \left[-\Lambda_{\infty}{ }^{2}(1-\cos \psi)^{2}\right]\right\}^{1 / 2} .
\end{gathered}
$$

This expression is exact along the singular ray $\cos \beta^{*}=1$, where it reduces to 


$$
\begin{aligned}
\frac{q_{\infty}}{W_{\infty}}=\frac{\pi}{2}(\mathrm{Kn})^{-1}\left\{( 1 + \frac { u _ { M } } { u _ { \infty } } ) \operatorname { e x p } \left[\frac{-\Lambda_{\infty}{ }^{2}}{2}\right.\right. & \left.(1+\cos \psi)^{2}\right] \\
& \left.+\left|1-\frac{u_{M}}{u_{\infty}}\right| \exp \left[\frac{-\Lambda_{\infty}^{2}}{2}(1-\cos \psi)^{2}\right]\right\} .
\end{aligned}
$$

Along the singular ray, the hypersonic approximation breaks down for large $y$, since the boundary condition at infinity requires $q_{\infty}$ to vanish there. Actually, unscattered molecules arriving from directions other than that of the freestream flow reach the ray $\theta=\psi, \phi=0$ for large $y$, permitting the boundary condition to be satisfied. Along any adjacent ray corresponding to a fixed value of $\cos \beta^{*}$ such that $\cos \beta^{*} \neq 1$, when $(\mathrm{Kn} / y)^{2}$ is sufficiently small compared with $1-\cos ^{2} \beta^{*}$, (14) reduces to

$$
\begin{aligned}
\frac{q_{\infty}}{W_{\infty}} \cong \frac{\pi}{2 y}\left(1-\cos ^{2} \beta^{*}\right)^{1 / 2}\left\{\left(1+\frac{u_{M}}{u_{\infty}}\right) \exp [\right. & \left.-\Lambda_{\infty}{ }^{2}(1+\cos \psi)^{2}\right] \\
\left.+\left|1-\frac{u_{M}}{u_{\infty}}\right| \exp \left[-\Lambda_{\infty}{ }^{2}(1-\cos \psi)^{2}\right]\right\} &
\end{aligned}
$$

Thus, the boundary condition at infinity is recovered, but with increasing slowness as the singular ray is approached. Note that the quantity $y\left(1-\cos ^{2} \beta^{*}\right)^{1 / 2}$ is proportional to the distance in the plane perpendicular to the ray $\cos \beta^{*}=1$ from the ray to the point in question. The "shadow" region is then a cylindrical tube surrounding the singular ray. Since the inhomogeneous term $W_{\infty}^{-2} H_{4}\left(W_{\infty}, q_{\infty}\right)$ is roughly an exponential in $\left(-q_{\infty} / W_{\infty}\right)$, the width of the shadow region is given (in units $\left.(B A)^{1 / 2} \mathrm{Kn}^{-1}\right)$ by the factor multiplying $y^{-1}\left(1-\cos ^{2} \beta^{*}\right)^{-1 / 2}$ in (16).

Now consider the homogeneous term in (11). The variable $q_{0}=\left(m / k T_{0}\right) \tau^{*}$ has the explicit form:

$$
\begin{aligned}
& \frac{q_{e}}{W_{e}\left(y^{\prime}\right)}=(\mathrm{Kn})^{-1} \frac{u_{\infty}}{u_{e}\left(y^{\prime}\right)} \exp \left\{-\frac{1}{2} \Lambda_{\infty}{ }^{2}(1-\cos \theta)^{2}\right\}\left[1+\frac{u_{M}{ }^{2}}{u_{\infty}}-2 \frac{u_{M}}{u_{\infty}} \cos \beta^{*}\right]^{1 / 2} \\
& \quad \cdot\left\{\tan ^{-1}\left[\frac{y}{\mathrm{Kn}} \exp \frac{1}{2} \Lambda_{\infty}{ }^{2}(1-\cos \theta)^{2}\right]-\tan ^{-1}\left[\frac{y^{\prime}}{\mathrm{Kn}} \exp \frac{1}{2} \Lambda_{\infty}{ }^{2}(1-\cos \theta)^{2}\right]\right\} .
\end{aligned}
$$

The quantity $q_{e}$ is proportional to the number of collisions experienced by a molecule initially penetrating to a distance $y^{\prime}$ along a given radial ray as it travels outward along the ray to the field point at $y$. Note that $q_{e} / W_{e}\left(y^{\prime}\right)$ becomes very large $\left(O\left(\mathrm{Kn}^{-1}\right)\right.$ for $\theta<60^{\circ}$ and realistic values of $\Lambda_{\infty}$ and $\left.u_{\infty} / u_{0}\right)$ at $y^{\prime}=0$ for fixed $y$. Since the function $H_{3}\left(W_{e}, q_{e}\right)$ is roughly exponential in $\left(-q_{e} / W_{e}\right)$, the probability that those few molecules deposited upstream of the exit can penetrate to the other side is very small. Moreover, the contribution to the density at any point from molecules scattered radially inward towards the nozzle is exponentially smaller in $W_{e}{ }^{2}$ than that from molecules scattered radially outward away from the nozzle. For these reasons, the lower limit of integration in the homogeneous term of (11) is the origin. Similarly, the overwhelming preponderance of outward scattering events means that there is no contribution to the number density at $y$ from points $y^{\prime}>y$ on that ray. Further details are given in Appendix A. Finally, the functions $H_{n}$ may be simplified by applying the method of steepest descent once again. The result, valid for $p \gg 1$ and $Z \equiv q / p^{3}$ finite, is the first term in Anderson and Macomber's [2] asymptotic expansion: 


$$
\begin{aligned}
H_{n}(P, q) & =\frac{\left(p t_{0}\right)^{n-2}}{\left(1+2 Z / t_{0}^{3}\right)^{1 / 2}} \exp \left\{\frac{-p^{2} Z}{t_{0}}\left[1+\frac{1}{2} \frac{Z}{t_{0}^{3}}\right]\right\}, \\
t_{0} & =\left\{\frac{1}{3}+\frac{Z}{2}+\frac{1}{27}+\left(\frac{Z}{27}+\frac{Z^{2}}{4}\right)^{1 / 2}\right\}^{1 / 3}+\left\{\frac{Z}{2}+\frac{1}{27}-\left(\frac{Z}{27}+\frac{Z^{2}}{4}\right)^{1 / 2}\right\}^{1 / 3}, \\
Z & =q / p^{3} .
\end{aligned}
$$

Note that as $q \rightarrow 0, H_{n}(p, 0) \cong p^{n-2}$, while for large $q>0$

$$
H_{n} \sim \frac{q^{(n-2) / 3}}{(3 / 2)^{1 / 2}} \exp \left\{-\frac{3}{2} q^{2 / 3}\right\}
$$

The quantity $p^{2} Z$ in the exponential is equal to $q_{\infty} / W_{\infty}$ in the inhomogeneous term of (11), and to $q_{e} / W_{e}$ in the homogeneous term. These quantities are independent of $W_{\infty}$ and $W_{e}$, respectively. Thus, the quantity $W_{\infty}$ and $W_{e}$ enter the $H_{n}$ functions only through the quantity $Z$, which is formally $O\left(W_{\infty}^{-2}\right)$ or $O\left(W_{e}^{-2}\right)$ for fixed $y$. These terms are retained in (18) to allow $y$ to vary from a moderate multiple of $\mathrm{Kn}$ to values $\gg 1$. The principal effect of this variation is to shift the location of the saddle point $t_{0}$ for radius of $y \leq 1 / 2$. Although the shift is not large, the presence of the quantity $t_{0}$ in the exponent of (18) can change the computed value of $n / n_{\infty}$ at a given location by as much as a factor of two.

The physical picture represented by (11) may be summarized as follows. Molecules entering in a beam parallel to the undisturbed flow are scattered out of the beam by the exhaust gas, which is unaffected by the process. The scattered molecules are thus deposited along each radial ray in accordance with the inhomogeneous term in (11). The molecules are then caught up in the radial expansion and swept away from the nozzle, undergoing additional collisions as they proceed radially outward. The speed ratio of both the incident beam and the scattered gas is so high that the thermal spread in molecular velocities may be ignored, in comparison with the velocities considered. The exhaust gas density is so high in the vicinity of the nozzle exit that no molecules can penetrate this region. As the radial distance from the nozzle exit increases, the penetration process becomes more efficient. Ultimately, the exhaust gas density is sufficiently low for the ambient molecules to pass through the plume undisturbed, and the ambient density is recovered. The phenomena outlined above are illustrated schematically in Fig. 3.

The remainder of this paper deals with the solution of (11), and the computation of two examples. The following section contains an asymptotic, analytic solution of this equation, valid far from the nozzle exit. Sec. 5 discusses the computational procedures employed in the direct numerical solution of (11) and in the evaluation of the asymptotic result of Sec. 4. The analytical and numerical methods explained in these two sections are applied to an axially symmetric flow $(\psi=0)$ and a nonsymmetric, "side-blowing" flow $\left(\psi=90^{\circ}\right)$ in Sec. 6.

4. The asymptotic solution. When the radial coordinate $y$ is $O(1)$, an approximate solution to (11) may be readily obtained. Under these circumstances, $u_{e}\left(y^{\prime}\right)$ approaches $u_{M}$, while $q_{e} / W_{e}$ becomes

$$
\frac{q_{e}}{W_{e}} \cong \frac{u_{\infty}}{u_{M}}\left[1+\left(\frac{u_{M}}{u_{\infty}}\right)^{2}-2 \frac{u_{M}}{u_{\infty}} \cos \beta^{*}\right]^{1 / 2} \exp \left[-\Lambda_{\infty}^{2}(1-\cos \theta)^{2}\right]\left(\frac{1}{y^{\prime}}-\frac{1}{y}\right) .
$$




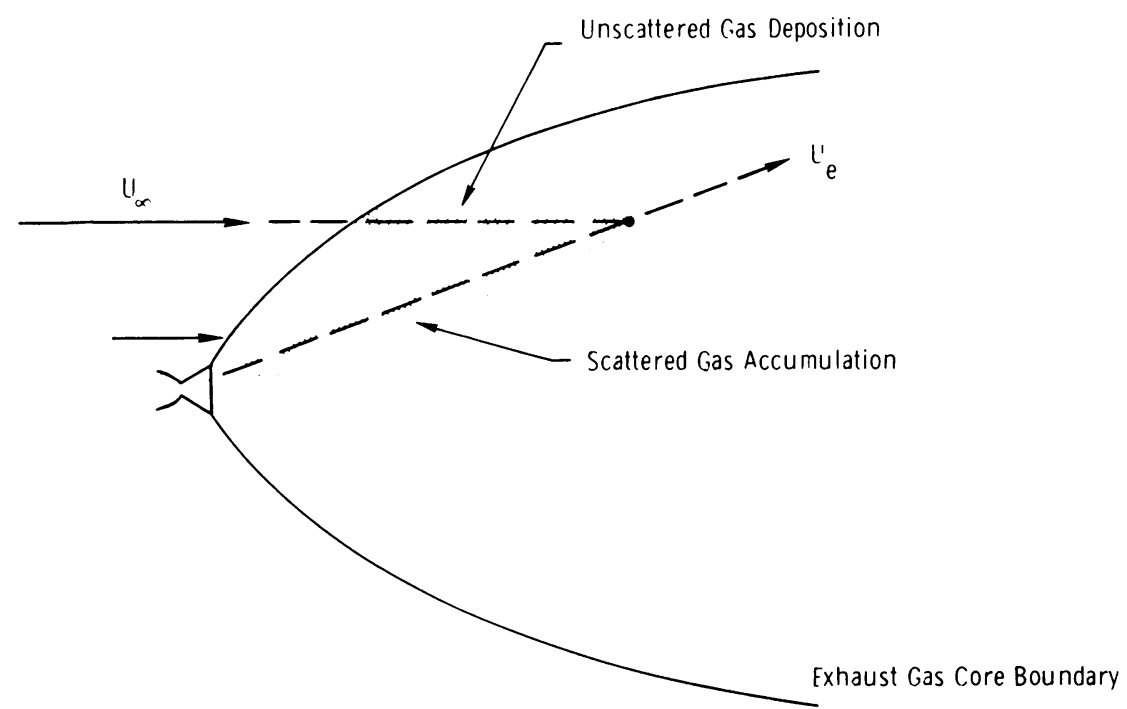

FIG. 3. Schematic of atmospheric penetration process.

Thus, $q_{e} / W_{e}$ is $O(1)$, which means that the quantity $Z \equiv q_{0} / W_{e}{ }^{3}$ in the function $H_{3}$ may be set equal to zero, reducing $H_{3}$ to an exponential. Eq. (11) then reduces to

$$
\begin{gathered}
{\left[\frac{n}{n_{\infty}}-\frac{1}{W_{\infty}^{2}} H_{4}(y)\right] J(y)=\int_{0}^{\nu} d y^{\prime} G\left(y^{\prime}\right) \frac{n}{n_{\infty}}\left(y^{\prime}\right),} \\
J(y)=y^{2} \exp \left\{-\frac{u_{\infty}}{u_{M} y}\left[1+\left(\frac{u_{M}}{u_{\infty}}\right)^{2}-2\left(\frac{u_{M}}{u_{\infty}}\right) \cos \beta^{*}\right]^{1 / 2} \exp \left[-\Lambda_{\infty}{ }^{2}(1-\cos \theta)^{2}\right]\right\}, \\
G\left(y^{\prime}\right)=\frac{J(y)}{y^{2}} \frac{u_{\infty}}{u_{M}}\left[1+\left(\frac{u_{M}}{u_{\infty}}\right)^{2}-2 \frac{u_{M}}{u_{\infty}} \cos \beta^{*}\right]^{1 / 2} \exp \left[-\Lambda_{\infty}{ }^{2}(1-\cos \theta)^{2}\right] .
\end{gathered}
$$

Eq. (20) is readily solved by differentiating with respect to $y$ to obtain a first-order differential equation for the quantity $\left[n / n_{\infty}-1 / W_{\infty}^{2} H_{4}(y)\right] J(y)$. Examination of (20), as $y$ approaches zero, shows that this quantity should vanish at the origin. Thus

$$
\left[\frac{n}{n_{\infty}}-\frac{1}{W_{\infty}^{2}} H_{4}(y)\right]=\frac{u_{\infty}}{y^{2} u_{M}} \exp \left[-\Lambda_{\infty}^{2}(1-\cos \theta)^{2}\right] \int_{0}^{\nu} d y^{\prime} \frac{1}{W_{\infty}^{2}} H_{4}(y) .
$$

The function $H_{4}(y)$ can be simplified in a similar manner away from the singular ray. The function $q_{\infty}$ then has the form:

$$
\begin{aligned}
q_{\infty} / W_{\infty} & \cong \Omega / y, \\
\Omega & =\int_{-\infty}^{0} d t \frac{\left[1+\left(u_{M} / u_{\infty}\right)^{2}-2\left(u_{M} / u_{\infty}\right) \cos \delta\right]^{1 / 2} \exp \left[-\Lambda_{\infty}{ }^{2}(1-\cos \mu)^{2}\right]}{1+t^{2}+2 t \cos \beta^{*}} .
\end{aligned}
$$

Then, provided that the numerical value of $\Omega$ on the ray under consideration is not too large, $q_{\infty} / W_{\infty}^{3}$ may be set equal to zero in the function $\left(1 / W_{\infty}{ }^{2}\right) H_{4}\left(W_{\infty} q_{\infty}\right)$, thereby reducing it to an exponential in $(-\Omega / y)$. The quadrature on the right-hand side of $(21)$ may then be evaluated in terms of the exponential integral function $E_{2}(\zeta)$, yielding the result: 


$$
\begin{gathered}
\frac{n}{n_{\infty}}=\frac{1}{W_{\infty}^{2}} H_{4}\left(W_{\infty}, q_{\infty}\right)+\frac{u_{M}}{u_{M} y}\left[1+\left(\frac{u_{M}}{u_{\infty}}\right)^{2}-2\left(\frac{u_{M}}{u_{\infty}}\right) \cos \epsilon\right]^{1 / 2} \\
\cdot \exp \left[-\Lambda_{\infty}^{2}(1-\cos \theta)^{2}\right] \cdot E_{2}\left(\frac{\Omega}{y}\right), \\
E_{2}(\zeta)=\int_{1}^{\infty} \frac{\exp (-\zeta t)}{t^{2}} d t
\end{gathered}
$$

The first term in the solution (23) could also formally be replaced by $\exp \{-\Omega / y\}$, to the present order of accuracy. However, the analysis in this section can be interpreted as an attempt to find an approximate expression for the scattered gas contribution to the number density, as represented by the homogeneous term in (11). The second term in the solution (23) is the desired result, as is the right-hand side of (21). However, the latter expression is too complicated to study analytically, and sufficiently expensive in computer time (relative to the direct numerical solution of (11)) not to warrant its use without the further approximation represented by (23).

Now consider the behavior of the solution as a function of $y$ and $\theta$. For small $y$ (assuming that the asymptotic solution is at least qualitatively correct), the function $E_{2}(\Omega / y)$ has the asymptotic expansion

$$
E_{2} \sim \frac{y \exp (-\Omega / y)}{\Omega}\{1+O(\Omega / y)\}
$$

The scattered gas contribution becomes very small as the nozzle exit is approached because few ambient molecules are able to penetrate the dense region of the plume. For large angles $\theta$, the factor $\exp \left\{-\Lambda_{\infty}^{2}(1-\cos \theta)^{2}\right\}$ (representing the angular dependence of the exhaust gas density distribution) cuts off the scattered gas contribution because there are so few exhaust gas molecules to serve as scatterers. For large $y, E_{2}(\Omega / y)$ may be approximated by $E_{2} \sim(\Omega / y) \log (\Omega / y)$. Thus, the scattered gas density rises to a peak, then decays much more slowly with increasing $y$. The decay is caused by the increasing volume in the plume available to molecules scattered much nearer to the nozzle and then convected radially outward. The additional local scattering far from the nozzle contributes weakly, accounting for the logarithmic factor in the scattered result. However, the exhaust gas density ultimately decreases to the point where the unscattered contribution dominates, permitting the uniform boundary condition at infinity to be recovered.

5. The numerical computation procedure. The numerical computation of $n / n_{\infty}$ requires the evaluation of the inhomogeneous term in (11) or (23) when the asymptotic solution is valid, together with a procedure for calculating the homogeneous term in the integral equation. The evaluation of the asymptotic approximation to the $H_{n}$ functions is straightforward, as is the computation of the function $E_{2}$ as a function of its argument. Hence attention is confined to the computation of $q_{\infty}$, which determines the value of the inhomogeneous term, and to the evaluation of the homogeneous term in (11).

The computation of the integral defining $q_{\infty}$, as given by (13), will be considered first. Note that the integrand is not singular in the entire range of integration, since its denominator is equal to or greater than one throughout this range. However, as shown in Sec. 3, the limit of $q_{\infty}$ as $y \rightarrow \infty$ and $\cos \beta^{*} \rightarrow 1$ is nonuniform; that is, the value of the limit depends on the order in which these two limiting processes take place. Numerically, 
this nonuniformity means that one must be very careful in evaluating this integral when both $y$ is $O(1)$ or larger and $\cos \beta^{*}$ is very close to 1 . For such values of $y$ and $\cos \beta^{*}$, most of the contributions to the integral come from points very close to $t=-1$, and the accuracy with which $\cos \delta$ and $\cos \mu$ can be evaluated at these points is limited by the arithmetical precision of a computer.

A close examination of the variation of the integrand $J$ in (13) with respect to $t$ shows that the integrand varies smoothly in the entire range of integration, so long as $\cos \beta^{*}$ is not close to 1 . For $\cos \beta^{*}$ near 1 , the integrand has very sharp variations near $t=-1$, and a peak value in that neighborhood. For $\cos \beta^{*}=1$, the peak of the integrand is exactly at $t=-1$, and is discontinuous.

In view of the above described peculiarities at the integrand $J$ of (13), the following method was devised to compute this integral. The integral, $I$, is divided into two parts, $I_{L}$ and $I_{R}$ :

$$
\begin{aligned}
I & =I_{L}+I_{R}, \\
I_{L} & =\int_{-\infty}^{-1-\epsilon_{1}} J(t, y) d t, \quad I_{R}=\int_{-1+\epsilon_{2}}^{0} J(t, y) d t .
\end{aligned}
$$

where $\epsilon_{1}$ and $\epsilon_{2}$ are two small positive numbers. $\epsilon_{1}$ is chosen such that the omitted part of the integral, from $-1-\epsilon_{1}$ to -1 divided by $I_{L}$, is equal to the specified fractional tolerance (TOL) for the computation of the inhomogeneous and homogeneous terms. $\epsilon_{2}$ is chosen in a similar fashion. It can be shown that, for $\cos \beta^{*}=1, \epsilon_{1}$ and $\epsilon_{2}$ are given by

$$
\begin{aligned}
& \epsilon_{1}=\frac{\pi}{2} \text { TOL } \exp \left(-\frac{\Lambda_{\infty}{ }^{2}}{2}(1+\cos \psi)^{2}\right) \frac{K n}{y} \\
& \epsilon_{2}=\text { TOL } \exp \left(-\frac{\Lambda_{\infty}{ }^{2}}{2}(1-\cos \psi)^{2}\right) \arctan \left[\frac{K n}{y} \exp \left(\frac{\Lambda_{\infty}{ }^{2}}{2}(1-\cos \psi)^{2}\right)\right] .
\end{aligned}
$$

Eqs. (24) are, in fact, used to compute $\epsilon_{1}$ and $\epsilon_{2}$ for any value of $\cos \beta^{*}$. It can be shown that these values of $\epsilon_{1}$ and $\epsilon_{2}$ are smaller than the values required for $\cos \beta^{*} \neq 1$, thus resulting in smaller values for the omitted parts of the integral.

$I_{L}$ is computed in steps $\Delta t$ of the integration variable $t$, starting from $t=-1-\epsilon_{1}$, and advancing to the left towards $t=-\infty$. The magnitude of each step $\Delta t$ is determined so that the integrand changes by about one order of magnitude in the range of the step. The contribution to $I_{L}$ from each step is computed through a six-point Gaussian integration. $I_{R}$ is computed in a similar, steplike fashion, starting from $t=-1+\epsilon_{2}$ and advancing to the right towards the origin. $I_{L}$ and $I_{k}$ are computed concurrently, so that the total integral $I$ is progressively built up from both sides of the point $t=-1$. The computation of $I_{L}$ is terminated when the most recent contribution to $I_{L}$, divided by the accumulated $I$, is less than the fractional tolerance, TOL, of the computation. The computation of $I_{R}$ is terminated in a similar fashion, or when the origin is reached.

Now consider the calculation of the homogeneous term in the integral equation. For computational purposes, it is more convenient to work with the radial coordinate $r^{*}$, defined by

$$
r^{*} \equiv r /(B A)^{1 / 2}=(\mathrm{Kn})^{-1} y .
$$

The initial growth of the homogeneous term in this variable is slow and well-behaved, taking place over the range $10<r^{*}<100$ along most radial rays. Letting the dimen- 
sionless, inhomogeneous and homogeneous quantities in (11) be $n_{i}{ }^{*}$ and $n_{h}{ }^{*}$, respectively, this equation may be written in the form:

$$
\begin{aligned}
n_{h}\left(r^{*}\right) & =\int_{r_{1} *}^{r^{*}} \tilde{K}\left(r^{\prime}, r^{*}\right) n^{*}\left(r^{\prime}\right) d r^{\prime}, \\
n^{*}\left(r^{\prime}\right) & =n_{i}^{*}\left(r^{\prime}\right)+n_{h}{ }^{*}\left(r^{\prime}\right) .
\end{aligned}
$$

In the "exact" definition of the integral equation, $r_{1}{ }^{*}$ is equal to zero. However, in the numerical evaluation of the integral of this equation, $r_{1}{ }^{*}$ can be much larger than zero, as will be shown later.

Now consider the following mesh of points:

$$
r_{1}{ }^{*}, r_{2}{ }^{*}, r_{3}{ }^{*} \cdots r_{m-2}{ }^{*}, r_{m-1}{ }^{*}, r_{m}{ }^{*}, r_{m+1}{ }^{*}, r_{m+1}{ }^{*}=r_{m}{ }^{*}+h_{m} .
$$

Note that, in general, a variable mesh size is assumed. Assume that the values of $n^{*}$ are known at all points up to and including the point $r_{m}{ }^{*}$. The value of $n_{h}{ }^{*}$ at the next point, $r_{m+1}{ }^{*}$, is computed by the following procedure.

The integral of (26) is divided into two parts, so that

$$
n_{h}^{*}\left(r_{m+1}^{*}\right)=I_{1}+I_{2},
$$

where

$$
\begin{aligned}
& I_{1}=\int_{r_{m^{*}}}^{r_{m+1} *} \tilde{K}\left(r^{\prime}, r_{m+1} *\right) n^{*}\left(r^{\prime}\right) d r^{\prime}, \\
& I_{2}=\int_{r_{1} *}^{r_{m^{*}}^{*}} \tilde{K}\left(r^{\prime}, r_{m+1}^{*}\right) n^{*}\left(r^{\prime}\right) d r^{\prime},
\end{aligned}
$$

$I_{1}$ is evaluated by the parabolic integration formula (B10) of Appendix B. Thus

$$
\begin{aligned}
I_{1}=-\frac{h_{m}{ }^{3}}{6 h_{m-1}\left(h_{m}+h_{m-1}\right)} K\left(r_{m-1}{ }^{*}, r_{m+1}{ }^{*}\right) n^{*}\left(r_{m-1}{ }^{*}\right)+\frac{h_{m}\left(3 h_{m-1}+h_{m}\right)}{6 h_{m-1}} \\
\cdot K\left(r_{m}{ }^{*}, r_{m+1}{ }^{*}\right) n^{*}\left(r_{m}{ }^{*}\right)+\frac{h_{m}\left(3 h_{m-1}+2 h_{m}\right)}{6\left(h_{m}+h_{m-1}\right)} \\
\cdot K\left(r_{m+1}{ }^{*}, r_{m+1} *\right)\left[n_{i}{ }^{*}\left(r_{m+1}{ }^{*}\right)+n_{h}{ }^{*}\left(r_{m+1}\right)\right] .
\end{aligned}
$$

Substituting $I_{1}$ from (32) in (29), and solving the resulting equation for $n_{h}{ }^{*}\left(r_{m+1}{ }^{*}\right)$, one obtains

$$
n_{h}{ }^{*}\left(r_{m+1}{ }^{*}\right)=\frac{I_{1}{ }^{\prime}+I_{2}}{1-\left[h_{m}\left(3 h_{m-1}+2 h_{m}\right) / 6\left(h_{m}+h_{m-1}\right)\right] \widehat{K}\left(r_{m+1}{ }^{*}, r_{m+1}{ }^{*}\right)}
$$

where $I_{1}{ }^{\prime}$ is equal to $I_{1}$, as given by (32), with $n_{h}{ }^{*}\left(r_{m+1}{ }^{*}\right)$ set to zero.

As can be seen from (31) and (32), the right-hand side of (33) involves

(i) The values of $n^{*}$ for all points up to and including the point $r_{n}{ }^{*}$, which are known; and

(ii) The value of $n_{i}{ }^{*}$ at the point $r_{m+1}{ }^{*}$ which can be evaluated separately. Thus, the right-hand side of (33) involves quantities that are known, or can be evaluated independently of $n_{h}{ }^{*}\left(r_{m+1}{ }^{*}\right)$; therefore, (33) is an explicit expression for the unknown $n_{h}^{*}\left(r_{m+1}^{*}\right)$.

$I_{2}$ is computed by successive applications of the parabolic integration formula (B11) 
of Appendix B to three points at a time, starting with the point $r_{m}{ }^{*}$ and advancing to the left towards the point $r_{1}{ }^{*}$. The reason for computing $I_{2}$ backwards, in this way, is that the kernel $\tilde{K}\left(r^{\prime}, r_{m+1}{ }^{*}\right)$ decreases sharply as $r^{\prime}$ becomes smaller and smaller than $r_{m}{ }^{*}$, and thus the contributions to $I_{2}$ decrease sharply. This means that the computation of $I_{2}$ can be terminated when a desired accuracy is achieved, much before reaching the initial point $r_{1}^{*}$. The computation of $I_{2}$ is terminated when the most recent contribution to $I_{2}$, divided by the sum of $I_{2}$ (accumulated) and $I_{1}{ }^{\prime}$, is smaller than a specified fractional tolerance (TOL), which is also the tolerance within which $n_{i}{ }^{*}$ is computed. If $m$ is even, and the computation of $I_{2}$ must be extended all the way to $r_{1}{ }^{*}$, then the last contribution to $I_{2}$ is from two points rather than three, namely points $r_{2}{ }^{*}$ and $r_{1}{ }^{*}$, and formula (B9) of Appendix B is applied instead of formula (B11).

The procedure described above for the computation of $n_{h}{ }^{*}$ requires that $n_{h}{ }^{*}$ be known at the first three points, $r_{1}{ }^{*}, r_{2}{ }^{*}$ and $r_{3}{ }^{*}$. The values of $n_{h}{ }^{*}$ at $r_{1}{ }^{*}$ is the initial condition of the problem, and when $r_{1}{ }^{*}$ is taken equal to zero, one has the "exact initial condition" of the problem, which is $n_{h}{ }^{*}(0)=0$. A close examination of the situation reveals that, as one travels along a radial ray from the origin outward, $n_{i}{ }^{*}$ decays rapidly from 1 to a very small value, then increases back to 1 , while $n_{h}{ }^{*}$ increases slowly from 0 to some maximum value, then decays back to zero. Thus, in the numerical evolution of $n_{h}{ }^{*}$, a great deal of computer time is saved, without any loss in accuracy, by starting the solution at some $r_{1}{ }^{*}$ larger than zero with the approximate initial condition $n_{h}{ }^{*}=0$ at $r_{1}{ }^{*} \cdot r_{1}{ }^{*}$ is selected so that it is in the region where $n_{i}{ }^{*}$ has its minimum plateau. The rapidly decaying kernel annihilates the errors associated with this approximate initial condition. Thus

$$
n_{h}^{*}\left(r_{1}^{*}\right)=0 .
$$

The value of $n_{h}{ }^{*}$ at $r_{2}{ }^{*}$ is computed by evaluating $I_{1}$ in (30) through the trapezoidal rule $\left(I_{2}\right.$ in this case is, of course, equal to zero). As for the value of $n_{h}{ }^{*}$ at $r_{3}{ }^{*}$, it is computed by evaluating both $I_{1}$ and $I_{2}$ in (30) and (31) by the trapezoidal rule. Thus

$$
\begin{gathered}
n_{h}{ }^{*}\left(r_{2}{ }^{*}\right)=\frac{h_{1} / 2\left[\tilde{K}\left(r_{1}{ }^{*}, r_{2}{ }^{*}\right) n^{*}\left(r_{1}\right)+\tilde{K}\left(r_{2}{ }^{*}, r_{2}{ }^{*}\right) n_{i}{ }^{*}\left(r_{2}{ }^{*}\right)\right]}{1-\left(h_{1} / 2\right) \tilde{K}\left(r_{2}{ }^{*}, r_{2}{ }^{*}\right)} \\
n_{h}{ }^{*}\left(r_{3}{ }^{*}\right)=\frac{\frac{1}{2}\left[h_{1} \tilde{K}\left(r_{1}{ }^{*}, r_{3}{ }^{*}\right) n^{*}\left(r_{1}{ }^{*}\right)+\left(h_{1}+h_{2}\right) \tilde{K}\left(r_{2}{ }^{*}, r_{3}{ }^{*}\right) n^{*}\left(r_{2}{ }^{*}\right)+h_{2} \tilde{K}\left(r_{3}{ }^{*}, r_{3}{ }^{*}\right) n_{i}{ }^{*}\left(r_{3}{ }^{*}\right)\right]}{1-\left(h_{2} / 2\right) \tilde{K}\left(r_{3}{ }^{*}, r_{3}{ }^{*}\right)} .
\end{gathered}
$$

If the integrating step $h_{m}$ from $r_{m}{ }^{*}$ to $r_{m+1} *$ is too large, then the denominator in (33) can be small, or even negative. This must not be allowed since instabilities will result. Now, it can be shown that:

$$
\tilde{K}\left(r_{m+1}{ }^{*}, r_{m+1}{ }^{*}\right)<\tilde{K}\left(r_{m}{ }^{*}, r_{m}{ }^{*}\right)
$$

and, therefore, if the integrating step is chosen so that

$$
h_{m}=\frac{\alpha_{1}}{\widetilde{K}\left(r_{m}{ }^{*}, r_{m}{ }^{*}\right)},
$$

the denominator of (33) is roughly equal to $1-0.5 \alpha_{1}$. Thus, for sufficiently small values of the parameter $\alpha_{1}$, instabilities can be avoided. The correct value of $\alpha_{1}$ for a given radial ray cannot be defined a priori, but one anticipates that a value between 0.1 
and 1 should do. Indeed, in the applications discussed in the next section, the value $\alpha_{1}=0.5$ was employed. This ambiguity in the right value of $\alpha_{1}$ notwithstanding, (37) and (38) show that $h_{m}$ increases as $r_{m}{ }^{*}$ increases, a very desirable result. A second criterion for the selection of $h_{m}$ is set up by fitting a parabola to the values of $n_{h}{ }^{*}$ at the three points $r_{m-2}{ }^{*}, r_{m-1}{ }^{*}$, and $r_{m}{ }^{*}$ and then extrapolating to a point $r_{m+1}{ }^{*}$ where the fractional change in $n_{h}{ }^{*}$ is a small number $\beta_{1}$, on the order of 0.01 to 0.1 . The value of $\beta_{1}$ actually used is 0.05 . The value of $h_{m}$ derived from this criterion is compared to that given by (38), and the smaller of the two is selected. For the two initial integrations with the trapezoidal rule, a sufficiently small integrating step is taken, readily satisfying the stability considerations discussed above.

In the terminology of numerical computation, the method described above for the computation of the homogeneous term in the integral equation would be classified as a second-order, variable multistep, direct, implicit method. Watanabe [9] has described and classified a variety of numerical methods for the solution of Voltera-type integral equations arising from kinetic theory problems. Note that the present method, although an implicit method, gives the result explicitly. This is possible because the integral equation is linear. The integral equations considered by Watanabe are nonlinear. Thus, the implicit methods that he describes involve either iterative or predictor-corrector techniques. There are two other basically new features in the present method, which contribute immensely to the efficiency and stability of the numerical computation. The first feature is the use of variable integrating steps selected locally. The second feature is the backward integration of the integral equation, from the field point $r_{m+1}$ * towards the origin, which exploits the sharp decay of the kernel away from $r_{m+1}{ }^{*}$. Apparently, this property of many kinetic theory kernels has not been exploited before; methods reported in the available literature invariably use a forward integration (from the origin to $\left.r_{m+1}^{*}\right)$.

A computer program in FORTRAN IV has been developed, employing the numerical procedures described above, and this program has been run successfully on the UNIVAC 1108 system. Before final production runs were made, the accuracy, stability, and efficiency of the program were investigated through several test runs, by use of a trial integral equation whose exact solution was known.

The procedure for calculating $n_{i}{ }^{*}$ works very well for any value of $r^{*}$, so long as $\cos \beta^{*}$ is not equal to 1 . The procedure for calculating $n_{h}{ }^{*}$ is stable and accurate. It is efficient for values of $\theta$ equal to or less than about $45^{\circ}$ if an accuracy of $0.1 \%$ (TOL $=$ 0.001 ) is required. By accepting an error of $0.5 \%$ (TOL $=0.005$ ), the program can be utilized out to angles $\theta$ of $60^{\circ}$. For larger values of $\theta$, the value of the parameter $\alpha_{1}$ required for an accurate solution is so small that the integrating steps become very small. Hence, a great deal of computer time is required to obtain the solution. For such values of $\theta, n_{h}{ }^{*}$ can be computed much more efficiently from the asymptotic expression (23), which is valid quite close to the origin for such large angles. A computer program for the evaluation of the asymptotic expressions for $n_{h}{ }^{*}$ has also been developed, and works quite well.

For an accuracy of one part in a thousand (TOL $=0.001$ ), it takes, on the average, about 30 sec of 1108 central processing unit (CPU) time to compute $n_{i}{ }^{*}$ and $n_{h}{ }^{*}$ along a radial ray, all the way from the origin to the value of $r^{*}$ where the ambient condition is recovered. The CPU time required for the calculation of $n_{h}{ }^{*}$ is about three times that for $n_{i}{ }^{*}$. 
6. Numerical results. The theoretical and numerical analyses described in the previous sections have been applied to the calculation of an axially symmetric flow ("aligned flow"), $\psi=0$; and to a "nonaligned" case, $\psi=90^{\circ}$. The values of the remaining parameters needed to determine uniquely the solution for $n / n_{\infty}$ were the same for both flows, and are listed in Table 1 . The integral equation was solved directly, to an accuracy of $0.1 \%$, on rays spaced at five-degree integrals for $\theta \leq 45^{\circ}$, and with a reduced accuracy of $0.5 \%$ for values of $\theta$ between $45^{\circ}$ and $60^{\circ}$. Eq. (23) was used to obtain results for angles $\theta$ greater than $60^{\circ}$.

TABLE 1. Parameters employed in computed results for $n / n_{\infty}$

$\begin{array}{cccccc}\mathrm{Kn} & W_{\omega} & u_{M} / u_{\infty} & \gamma & M_{0} & m / m_{e} \\ 10^{-3} & 6 & 0.3 & 1.3 & 4 & 0.8\end{array}$

First consider the aligned flow. Fig. 4 illustrates the relative magnitude of the scattered and unscattered contributions to the density on the $\theta=30^{\circ}$ ray. Near the nozzle exit, both contributions are small because most of the molecules in the incident, ambient stream are deposited before reaching the $30^{\circ}$ ray. As $y$ increases, more molecules are deposited on the ray, and then convected outward by the exhaust. Since the scattered gas at a given $y$ station is an accumulation of molecules scattered earlier, this buildup is quite rapid, and gives rise to the overshoot in density depicted in the figure. The combination of the geometric relieving effect and reduced scattering discussed in Sec. 4 then reduces the scattered gas contribution very slowly (note the logarithmic scale) to zero. The unscattered gas density rises monotonically with increasing $y$ to recover the boundary condition at infinity.

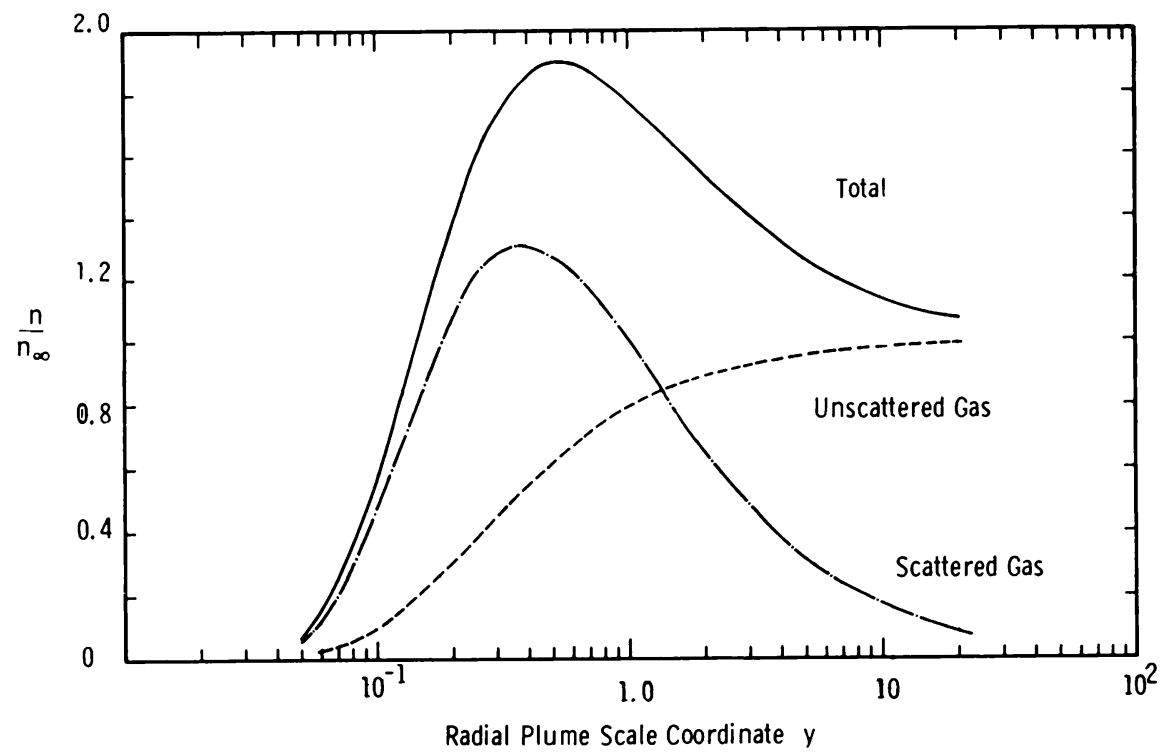

FIG. 4. Scattered and unscattered contribution to ambient penetration of exhaust plume $\left(\theta=30^{\circ}\right)$. 


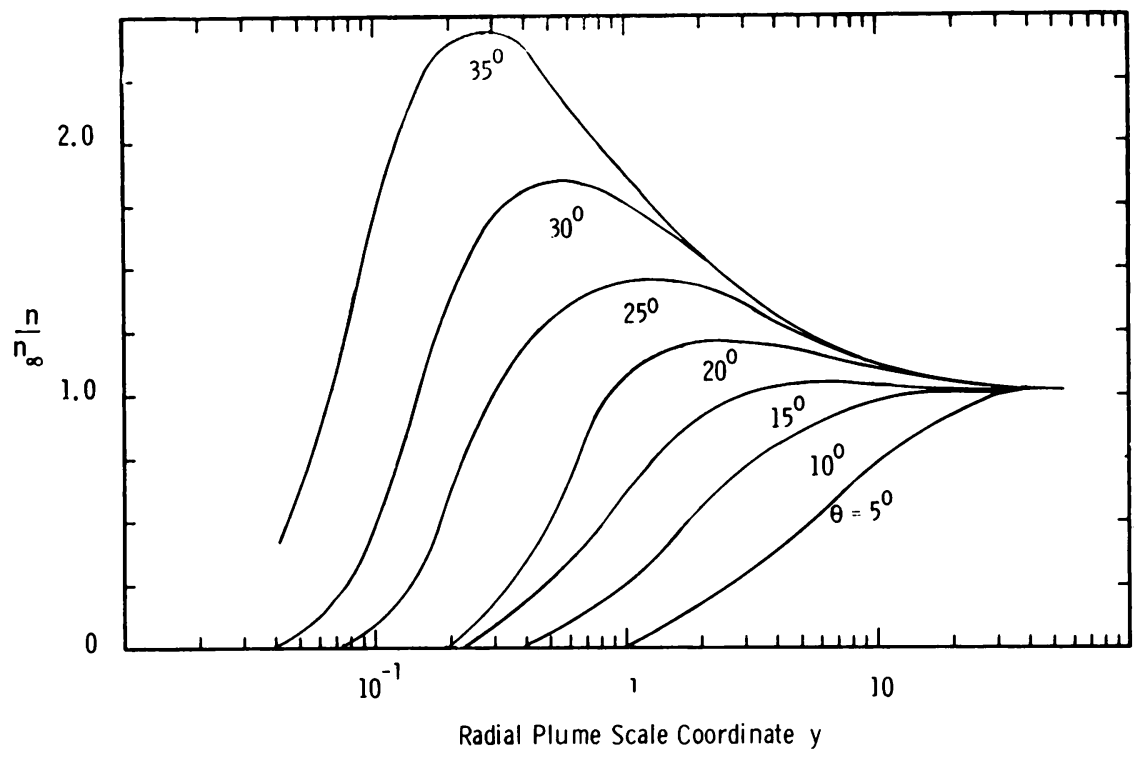

FIG. 5. Radial density distribution for aligned plume for values of $\theta=5^{\circ}$ to $35^{\circ}$.

The above picture holds qualitatively on all rays, with only the magnitude and location of the maximum in the scattered gas contribution changing from ray to ray. Figs. 5 and 6 show the development of the radial profiles with increasing $\theta$. Near the thrust axis, the recovery of the ambient condition is slowest because the plume is densest. The density overshoot begins at about $\theta=15^{\circ}$, and increases in magnitude with increasing $\theta$, until

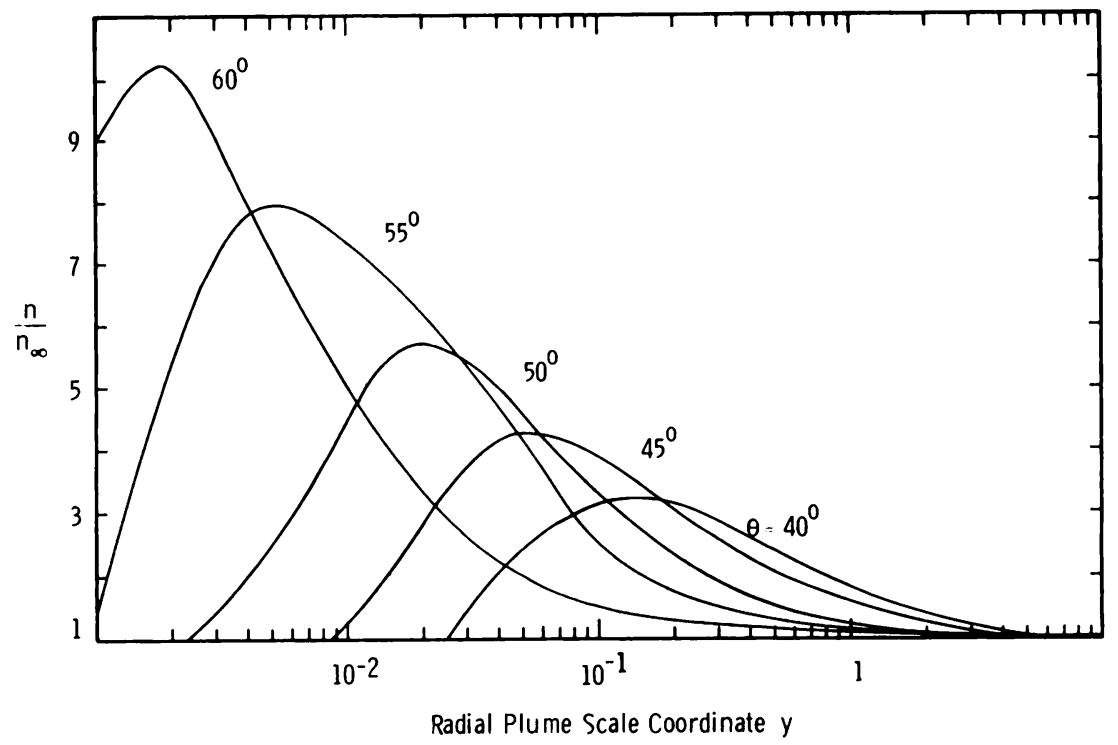

Fig. 6. Radial density distribution for aligned plume for values of $\theta=40^{\circ}$ to $60^{\circ}$. 
peak values are reached for values of $\theta$ slightly larger than $\theta=60^{\circ}$. The location of the peak moves closer to the nozzle with increasing $\theta$. For larger angles, the density drops rapidly towards the ambient value, because the exhaust gas density is so low that no significant scattering takes place.

The results predicted by the present theory for values of $y$ smaller than about $10^{-2}$, corresponding to $r^{*}=10$, must be treated with extreme caution. Since $r^{*}$ measures the radial coordinate in units of exit radii ( $B$ is about 0.3 in the present example), phenomena on this scale may be strongly influenced by the details of the geometry of the body carrying the motor. Moreover, even in the absence of a finite body, the exhaust gas characteristics in the vicinity of the nozzle are not described accurately by (2). However, the behavior of the solutions for larger $y$ is not sensitive to the results close to the nozzle exit.

The density is plotted as a function of angle at several radial stations in Fig. 7 . The ambient conditions are recovered progressively later in the expansion as $\theta$ decreases, since the region near the thrust axis is the least accessible to the atmospheric gases. Fig. 8 shows the corresponding curves for the windward portion of the plane containing the wind and thrust vectors in the nonaligned case. The density distribution is qualitatively similar to that in the aligned flow. However, the asymmetry is such that the recovery must be much slower on the leeward side (Fig. 9), because of the fact that the

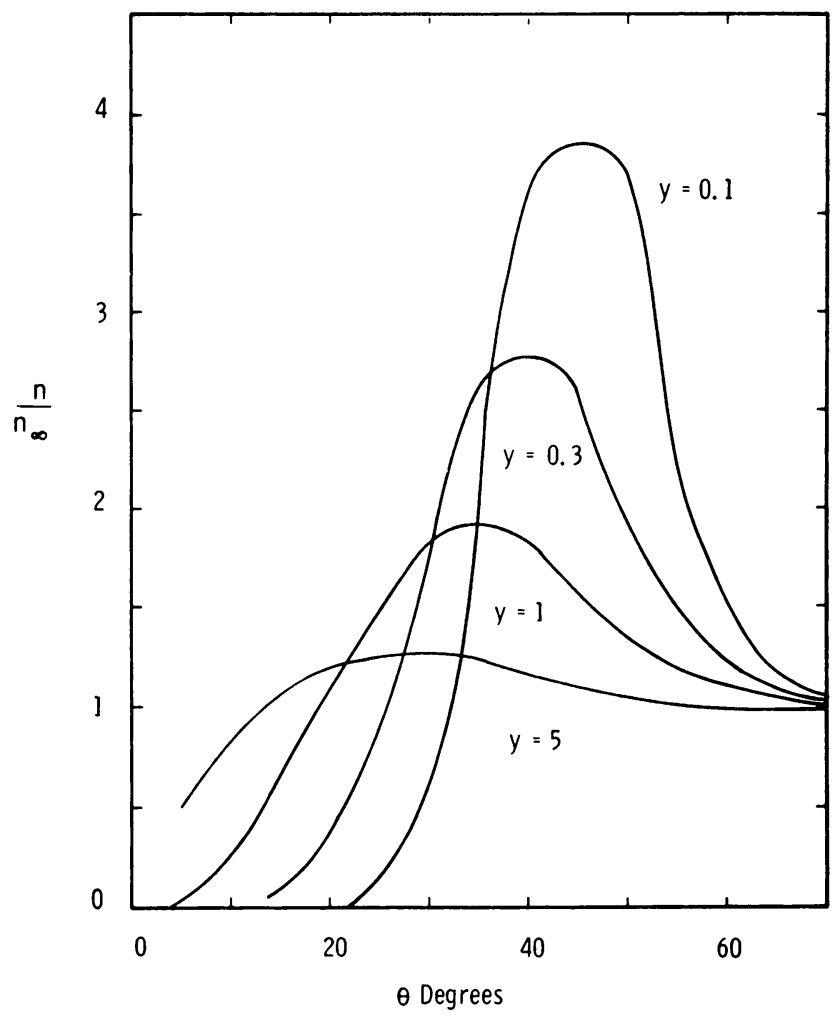

FIG. 7. Angular density distribution for aligned plume. 


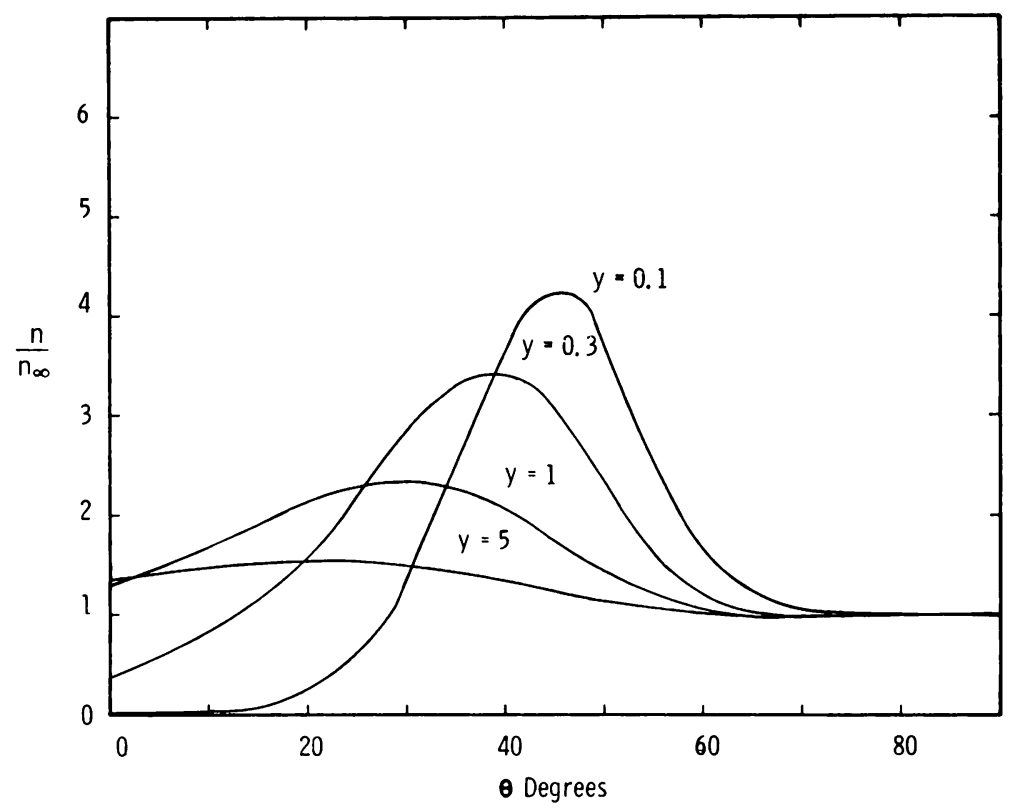

FIG. 8. Angular density distribution for nonaligned plume (windward side).

atmosphere must penetrate through the entire windward portion of the plume before reaching these rays. The singular ray is now at $\theta=90^{\circ}$, on the leeward side. Although the model predicts some scattering along this ray, the value of $q_{\infty} / W_{\infty}$ is $O\left(\exp \left(-\Lambda_{\infty}{ }^{2}\right) / K_{n}\right)$, which is less than $10^{-3}$ in the present problem.

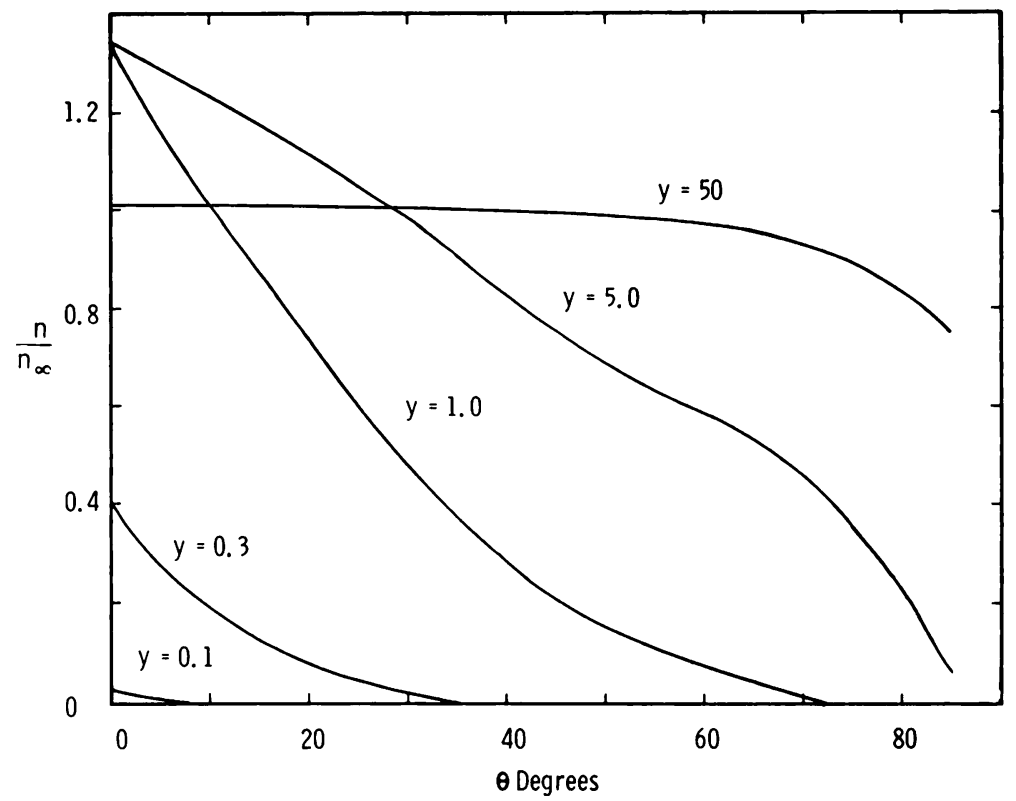

FIG. 9. Angular density distribution for nonaligned plume (leeward side). 
Appendix A. Hypersonic limit-homogeneous term. The homogeneous term in (7) may be rewritten (inverting the order of integration) as

$$
\begin{aligned}
\int_{-\infty}^{0} d s^{\prime} \frac{1}{2 \pi} \int_{0}^{2 \pi} d \eta \int_{0}^{\pi} \sin \xi d \xi\left(\frac{m}{k T_{e}}\right)^{1 / 2} \omega \\
\cdot \exp \left(-\frac{1}{2} W_{e}^{2}\left(1-\cos ^{2} \alpha\right)\right) H_{3}\left(W_{e} \cos \alpha, q_{e}\right) \frac{n}{n_{\infty}}, \\
q_{e}=\left(\frac{m}{k T_{e}}\right)^{1 / 2} \int_{e^{\prime}}^{0} d s \omega(\mathbf{x}+\hat{v} s) .
\end{aligned}
$$

Here, $\cos \alpha$ is given by (6), while the quantities $T_{\bullet}, \omega, W_{e}$, and $n / n_{\infty}$ are functions of $\mathbf{x}+\hat{v} s^{\prime}$. The basic assumption is analogous to that employed in Sec. 3 ; i.e., the dominant contribution to the angular integrations over $\xi$ and $\eta$ comes from the neighborhood of $\cos \alpha=1$, because of the large factor $W_{e}{ }^{2}$ in the exponential in the integrand of term (A1). By letting $x=\cos \xi$ be the first integration variable, the exponential factor in term (A1) becomes

$$
-\frac{1}{2} W_{e}^{2} \frac{r^{2}}{\left(\mathbf{x}+\hat{v} s^{\prime}\right)^{2}} f(x), \quad f(x)=1-\left[x \cos \theta+\left(1-x^{2}\right)^{1 / 2} \sin \theta \cos (\eta-\phi)\right]^{2} .
$$

Although the quantity multiplying $f(x)$ in (A2) is itself a function of $x$, through its dependence on the variable $\mathbf{x}+\hat{v} s^{\prime}$, the fact that it is large and positive for all values of $\hat{v}$ means that the contributions to (A1) will be exponentially small, except for values of $\hat{v}$ corresponding to $\cos \alpha=1$. Examination of (6) shows that $\cos \alpha=1$ can only occur for $\hat{v}=\hat{r}$. Thus, the exponential factor (A2) may be approximated by

$$
-\frac{1}{2} W_{e}^{2} \frac{r^{2}}{\left(r+s^{\prime}\right)^{2}} f(x) \text {. }
$$

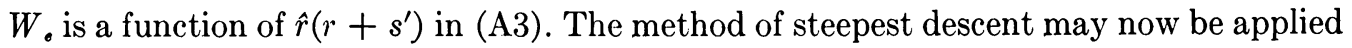
to (A1) in the same way that the corresponding integrals for the inhomogeneous term were evaluated in Sec. 3. The results implies that the remaining terms in the integral are to be evaluated at $x=\cos \theta, \eta=\phi$. When these values are inserted into expression (6), then $\cos \alpha=1$, as required for consistency, provided that $s^{\prime}+r$ is positive. Where $s^{\prime}+r$ is negative, $\cos \alpha=-1$, and the contribution to (A1) is again exponentially small in $W_{e}$, this time because of the fact that the function $H_{3}\left(-W_{e}, q_{e}\right)$ is $O\left(\exp \left(-W_{e}{ }^{2} / 2\right)\right)$. If, now, the substitution $r^{\prime}=s^{\prime}+r$ is made in the integration over $s^{\prime}$ which remains in (A1), then the homogeneous term reduces to that given in (11).

Appendix B. Parabolic integration formulae with variable integration step. Consider the following mesh of three points $x_{1}, x_{2}$, and $x_{3}: x_{1}, x_{2}=x_{1}+h_{1}, x_{3}=x_{2}+h_{2}$, and the following integrals of a function $f(x)$ :

$$
\begin{aligned}
& I_{1,2}=\int_{x_{1}}^{x_{2}} f(x) d x \\
& I_{2,3}=\int_{x_{2}}^{x_{3}} f(x) d x \\
& I_{1,3}=\int_{x_{1}}^{x_{3}} f(x) d x .
\end{aligned}
$$


Obviously,

$$
I_{1,3}=I_{1,2}+I_{2,3} \text {. }
$$

The objective here is to derive approximate expressions for these integrals, by use for $f(x)$ of the second-order Lagrange interpolating polynomial:

$$
\begin{aligned}
f(x)=\frac{f_{1}}{h_{1}\left(h_{1}+h_{2}\right)}\left(x-x_{2}\right)\left(x-x_{3}\right)-\frac{f_{2}}{h_{1} h_{2}}(x & \left.-x_{1}\right)\left(x-x_{3}\right) \\
& +\frac{f_{3}}{h_{2}\left(h_{1}+h_{2}\right)}\left(x-x_{1}\right)\left(x-x_{2}\right)
\end{aligned}
$$

where $f_{1}=f\left(x_{1}\right)$, and so on.

$$
\begin{aligned}
\int_{x_{1}}^{x_{2}}\left(x-x_{2}\right)\left(x-x_{3}\right) d x & =\frac{1}{3}\left(x_{2}{ }^{3}-x_{1}{ }^{3}\right)-\frac{1}{2}\left(x_{2}+x_{3}\right)\left(x_{2}{ }^{2}-x_{1}{ }^{2}\right)+x_{2} x_{3}\left(x_{2}-x_{1}\right) \\
& =\frac{1}{6}\left(x_{2}-x_{1}\right)^{2}\left(3 x_{3}-x_{2}-2 x_{1}\right)=\frac{h_{1}{ }^{2}}{6}\left(3 h_{2}+2 h_{1}\right) .
\end{aligned}
$$

By interchanging $x_{1}$ and $x_{2}$ in (B6), one obtains

$$
\begin{aligned}
\int_{x_{1}}^{x_{2}}\left(x-x_{1}\right)\left(x-x_{3}\right) d x & =-\frac{1}{6}\left(x_{1}-x_{2}\right)^{2}\left(3 x_{3}-x_{1}-2 x_{2}\right) \\
& =-\frac{h_{1}{ }^{2}}{6}\left(3 h_{2}+h_{1}\right) .
\end{aligned}
$$

By substituting $x_{2}$ for $x_{3}$ in (B7), one obtains

$$
\int_{x_{1}}^{x_{2}}\left(x-x_{1}\right)\left(x-x_{2}\right) d x=-\frac{1}{6}\left(x_{2}-x_{1}\right)^{3}=-\frac{h_{1}{ }^{3}}{6} .
$$

Therefore

$$
I_{1,2}=\frac{h_{1}\left(3 h_{2}+2 h_{1}\right)}{6\left(h_{2}+h_{1}\right)} f_{1}+\frac{h_{1}\left(3 h_{2}+h_{1}\right)}{5 h_{2}} f_{2}-\frac{h_{1}{ }^{3}}{6 h_{2}\left(h_{2}+h_{1}\right)} f_{3} .
$$

Furthermore, by interchanging $h_{2}$ and $h_{1}$, and $f_{1}$ and $f_{3}$ in (B9), one obtains

$$
I_{2,3}=-\frac{h_{2}{ }^{3}}{6 h_{1}\left(h_{2}+h_{1}\right)} f_{1}+\frac{h_{2}\left(h_{2}+3 h_{1}\right)}{6 h_{1}} f_{2}+\frac{h_{2}\left(2 h_{2}+3 h_{1}\right.}{6\left(h_{2}+h_{1}\right)} f_{3} .
$$

Finally, by adding (B9) and (B10), one obtains

$$
I_{1,3}=\frac{h_{2}+h_{1}}{6}\left[\frac{2 h_{1}-h_{2}}{h_{1}} f_{1}+\frac{\left(h_{2}+h_{1}\right)^{2}}{h_{1} h_{2}} f_{2}+\frac{2 h_{2}-h_{1}}{h_{2}} f_{3}\right] \text {. }
$$

The error involved in the approximate integration formulae (B9) to (B11) is of the order of $h^{4}$. For $h_{1}=h_{2}$, formulae (B9) and (B10) reduced to the well-known AdamsMoulton formula, while formula (B11) reduces to Simpson's rule.

\section{References}

[1] D. G. Anderson, On the steady Krook kinetic equation: part 1, J. Fluid Mech. 26, 17 (1966)

[2] D. G. Anderson and H. K. Macomber, Technical report 10, Engineering Sciences Laboratory, Harvard University (1964) 
[3] H. R. Baum, The interaction of a transient exhaust plume with a rarefied atmosphere, J. Fluid Mech. 58, 795 (1973)

[4] J. W. Brook and B. B. Hamel, Spherical source flow with a finite back pressure, Phys. Fluids 15, $1898(1972)$

[5] E. P. Gross and M. Krook, Model for collision process in gases: small amplitude oscillations of charged two-component systems, Phys. Rev. 102, 593 (1956)

[6] R. E. Grundy, Axially symmetric expansion of a monatomic gas from an orifice into a vacuum, Phys. Fluids 12, 2011 (1969)

[7] J. A. F. Hill and J. S. Draper, Analytical approximation for the flow from a nozzle into a vacuum, J. Spacecraft and Rockets 4, 1552 (1966)

[8] E. P. Muntz, B. B. Hamel and B. L. Maguire, Some characteristics of exhaust plume rarefaction, A. I. A. A. J. 8, 1651 (1970)

[9] D. S. Watanabe, Technical report 38, Engineering Sciences Laboratory, Harvard University (1971) 